AC 2011-1872: AERIAL PHOTOGRAPHY PROJECT USING MATLAB PROGRAMMING FOR FRESHMAN INTRODUCTION TO MECHANICAL ENGINEERING COURSE

Mario Gomes, Rochester Institute of Technology (COE) 


\section{Aerial photography project using MATLAB programming for freshman introduction to mechanical engineering course}

\section{Abstract}

A novel design/analysis project was developed and implemented for a freshman-level introduction to mechanical engineering course. One of the major objectives of the course was to introduce fundamental computer programming concepts using MATLAB. The project incorporated both creative freedom for teaching design methodology along with engineering analysis using simple engineering principles (Archimedes' principle, and geometric optics in this case). The design project also included the development of a semester long MATLAB computer program which was used to analyze the results of their design project (conducting an analysis of their best photograph of a target to determine the enclosed area). The materials for this project were chosen to be readily available and inexpensive. However, the level of analysis in the project had the potential to be very extensive if desired. The project provided a realistic application of the math and physics freshman engineering students have learned in high school and motivated the calculus that they will be learning their first year in their college engineering curriculum.

The design-build project was done in teams of four students. The materials provided were the same for each team of students and contained two identical disposable film cameras, along with other items. Each team was required to design and build a mechanical timer (from the set of given parts) which would autonomously trigger the camera shutter without any additional input from the users. This timer would then be taken aloft by a set of helium balloons of differing sizes which they could "purchase" using points on testing day. The students needed to determine a method for finding the height of the camera when aloft, calibrate the camera lenses on the ground, get their camera to their desired height using tethers, and have it take a photograph of the target area. Then analysis of the photograph would be done using the MATLAB program developed in stages during the course. The result of the analysis was to determine the area enclosed by the figure on the ground. Many different designs for timers were created from the materials given, ranging from water clock 
timers, viscous timers, ice timers, etc.

The programming component of the project was done as individuals, i.e. each student was responsible for writing the analysis software. The lab component of the course taught basic programming constructs such as loops, branching, functions, variable assignment, etc. After programming fundamentals were taught, a well defined section of the final program was written each week which incorporated those concepts. Ultimately, all the sections of the program were assembled and the final analysis software was finished. The software they wrote would take a scan of their photograph and allow them to estimate the area inside a marked off area on the ground using many rectangles (introductory Riemann sums). This project combined many of the core skills developed in the course into a cohesive goal.

\section{Introduction}

Freshman engineering design projects exist in many universities and their numbers have increased over the past few decades. For example, at Carnegie Mellon University, several open-ended freshman engineering design projects were created in each engineering department. ${ }^{1}$ The complexity, costs, and design of these projects varies widely between universities and also varies over time at the same university as well. However, in general most of these freshman design courses have the same objectives: to apply basic engineering principles to a project, build on concepts learned in their physics and math courses, to think creatively, to develop teamwork skills, to understand the the basics of the engineering profession. The design project described in this paper was created for undergraduate freshman mechanical engineering students. The pre-requisites for the course included high-school physics, algebra, geometry, and trigonometry. Calculus was not pre-requisite and a majority of the students had never had any exposure to calculus at the time of the course. A major goal in designing the project was to include the MATLAB programming part of the course as an integral component of the design project.

This course project has been implemented twice by the author. The first version of this project was designed for an introductory course in exploration (in Earth and Space Sciences) where students had the opportunity to take photographs of the surface of Mars using the THEMIS camera in orbit around Mars. This imaging project was the inspiration for designing the aerial photography project. Several changes were made from that course for the second iteration:

- Kits of materials were created for each team instead of a monetary budget for each team. This was done to reduce the amount of time required students to attain materials, to emphasize planning their designs before construction, and to increase student creativity. 


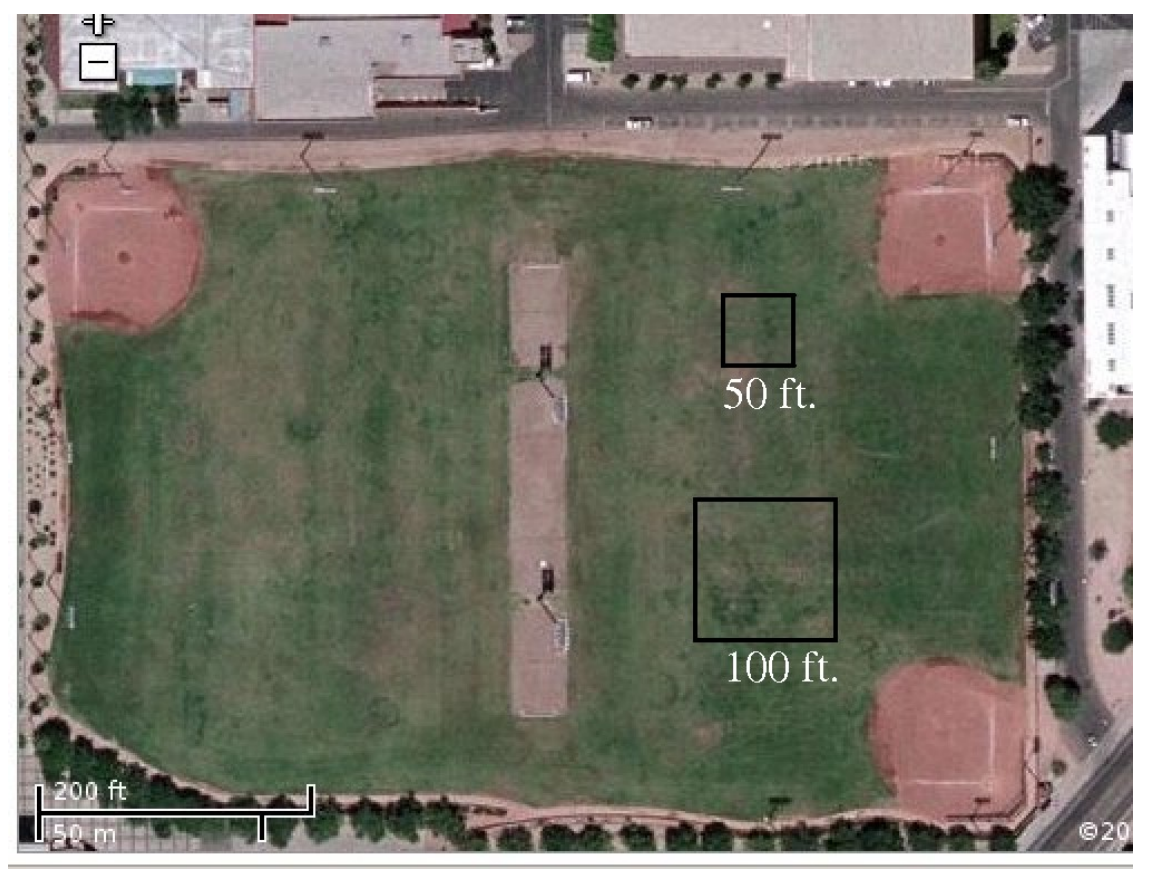

Figure 1: Upper and lower boundaries on test area size

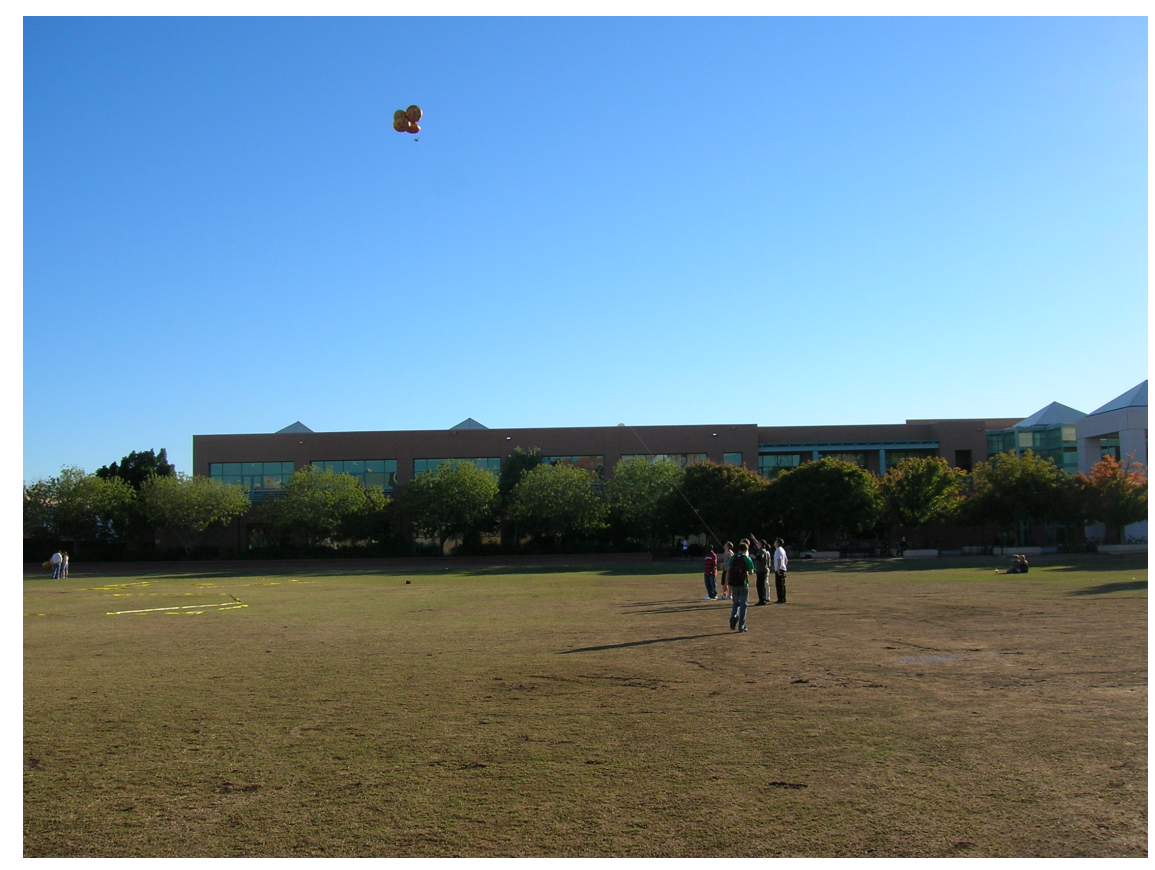

Figure 2: A student team taking photographic data on test day 
- Integration of Matlab programming as an integral component of the final project. Previously the Matlab programming project consisted of a 2D elliptical orbit simulation with a target on the surface of a circular rotating planet. Although this provided a challenging programming problem, a majority of students felt it was too difficult, and although it was closely linked to the THEMIS imaging project it did not directly relate to the design project.

- The target area now included a geometric shape with the goal of determining its enclosed area as opposed to comparing two photographs and looking for changes. This change was due, in part, to time constraints with an increased number of teams( 45 teams as opposed to 11 teams). A single set of photographs required less time for each team on final testing day. Also this change allowed the direct integration of the skills learned in the Matlab programming laboratory as a fundamental component of the design project.

\section{Project Rules (As given to the student teams)}

- Your materials budget for this project are the ones given to you in your "kit". You may ONLY use the given materials to make your trigger mechanism. You will need to get film developed into 4x6 inch prints. Pick one person in your team who is responsible for that. They will need to pay that cost, keep the receipt, and submit those receipts and a form to get reimbursed. Reimbursement takes a few weeks after the form has been received. IMPORTANT: Without a receipt you cannot get reimbursed.

- No flammable gases, liquids, or solids are allowed

- No open flames are allowed (i.e. burning paper fuses, etc.) Or anything else that has the potential to set fire to your device or the surrounding environment.

- Your design must return to ground in a safe manner (i.e. it must not pose a threat of bodily harm to any observers on the ground)

- All team members must maintain at least one foot on the ground at all times (i.e. no climbing up something to take pictures)

- You may not use any existing structures located on the test ground.

- No machining of parts is allowed. However, you may use hand tools (saws, files, etc.) and you are also allowed to use a power drill/drill press.

- Your device may not exceed an altitude of $500 \mathrm{ft}$.

- Your device must take your photos from at least 30ft. off the ground.

- No team members are allowed to be inside the target area when setting up or operating your device.

- Your camera must be triggered autonomously when airborne, i.e. you can't pull on a string to press the button. 
- You may "purchase" any number of balloons of any size from us on test day. However, you will only have 100 points with which to purchase balloons.

- Your team will have exactly 15 minutes on test day to take all the photos that you can, so practicing your approach and set-up before test day is necessary.

- You may only use a single photo for determining the size of target area.

- You may use at most 2 photos to determine the size of the SHAPE.

- You will be given(in your "kit") a sample piece of the string that we will provide for you on test day for a balloon tether. This string will not be marked and you will not be allowed to measure it on test day.

\section{Kit design}

Each team had the same kit of materials to work with. The kits were designed so that many types of mechanical timers could be constructed using the materials. Cost was a significant constraint on the kit design, however the materials developed for this kit were readily available and fairly inexpensive, especially when purchased in large quantities. 45 kits were constructed, one for each team of 3-5 students) and placed in team lockers in the work area for the students. The creation of the individual kits from the bulk materials was time consuming but only needed to be done once for the project. Although, none of the teams used all the materials, all of the materials were utilized amongst the entire class. The diversity of materials allowed for the design and construction of many different mechanical timers.

The student teams brainstormed many different trigger ideas and then consolidated these down to 3-5 ideas which they then sketched out in as much detail as they could. Informative and design sketching was heavily emphasized and revisions occurred at least twice during the project. A decision matrix with quantitative criteria was created to aid teams in reducing their choices to a single design. This design was then hand-drawn again in more detail and then constructed by the team in the shared working space using hand tools.

\section{Lift Calculations}

In order to determine the amount of lift required for the student designed camera and trigger payloads, a lift system had to be designed by the students. Although the option of building a kite was possible using the materials available in the students' kit, only a single student pursued this option (and only in the first year of the project, no team built a kite in the second revision of the project which is detailed here). Instead, as per the rules described above, student teams were given the option to "purchase" (using points) 


\begin{tabular}{|c|c|}
\hline Qty & description \\
\hline 1 & $500 \mathrm{ml}$ Water bottle \\
\hline 4 & Hot glue sticks \\
\hline 1 & 4oz bottle of Elmer's glue \\
\hline 1 & shop towel \\
\hline 2 & small wood blocks \\
\hline 12 & bamboo skewers \\
\hline 12 & very large rubber bands \\
\hline 2 & soda straws \\
\hline 4 & 7" zip-ties \\
\hline 2 & stirring straws \\
\hline 2 & wooden clothespins \\
\hline 4 & 6" latex balloons \\
\hline 1 & 9" latex balloon \\
\hline 1 & metal canning lid \\
\hline 1 & 10d common nail \\
\hline 2 & 16d finishing nails \\
\hline 6 & 4d finishing nails \\
\hline 1 & 30 gallon rip-stop plus garbage bag \\
\hline 1 & garbage bag twist tie \\
\hline 1 & small ziplock bag \\
\hline 1 & $1 / 4$ piece silly putty \\
\hline 2 & 800 ASA 27exp flash disposable camera \\
\hline $1 \mathrm{ft}$. & $12-3$ copper wire \\
\hline 1 & file folder \\
\hline 1 & $7 \frac{1}{2} \times 10$ mat board \\
\hline 1 & small tube superglue \\
\hline 2 & large IDEAL butterfly paperclips \\
\hline 3 & small IDEAL butterfly paperclips \\
\hline $6 \mathrm{ft}$. & sisal twine \\
\hline 3 & small regular paperclips \\
\hline 3 & sheets of coloured paper \\
\hline $10 \mathrm{in.}$ & aluminium foil \\
\hline 1 & small 18" round mylar balloon \\
\hline 1 & medium $25 "$ toroidal mylar balloon \\
\hline 1 & large 31" round mylar balloon \\
\hline 1 & mechanical hour timer \\
\hline $\begin{array}{r}3 \mathrm{ft} . \\
\text { yards }\end{array}$ & $\begin{array}{l}24 \text { guage flower wire } \\
\text { cotton thread }\end{array}$ \\
\hline $1 \mathrm{ft}$. & nylon button-thread \\
\hline
\end{tabular}

Table 1: Complete listing of contents of each team kit for the design project. Note that this kit includes 1 each of the mylar balloons(un-inflated) that could be "purchased" on test day. These mylar balloon samples were included in the kit so that volume estimates could be made and the weight of the balloon determined. 
helium filled balloons of various sizes on test day. Their kits provided a sample of each mylar balloon size which could then be used to determine the volume and weight of each balloon. Once an estimate of the volume and measurement of the weight of the balloon was found, the lift of each balloon could be predicted. Mylar balloons were used instead of latex balloons due to mylar's larger stiffness. The exercise of determining the lift of each of the three mylar balloons was given as an open-ended problem with no guidance outside of our class discussion of Archimedes' principle. Thus each team created their own method for determining the volume of the balloons and thus their lift. Some student teams chose to model the balloons as mathematical spheres and to experimentally measure a radius of each inflated balloon. Other teams chose to determine the volume experimentally by filling the balloon with water and measuring the amount of water required to fill the balloon. The mylar balloons were not strong enough to be filled with water and many of those teams resorted to estimating the volume by mathematical modeling. Many student teams chose to model the balloons as squat cylinders. However, they were free to choose their own models and some interesting models also emerged, for example, one team chose to model the balloon as a sphere $\left(4 / 3 \pi r^{3}\right)$ but then noticed that the Mylar balloon was rather squashed and they then chose to use a made-up formula $4 / 3 \pi r^{2} \cdot r_{z}$ and used that for the volume of the balloon. The students were not allowed access to helium so they could not measure the lift of the balloons directly. After their estimate were made and they had chosen which balloons they wanted to use on test day, we directly measured the lift of each balloon in class so they could see how accurate their estimates were.

Modeling the balloons:

small inflated dimensions: diameter $=14$ in., thickness $=5.5$ in.

small volume $($ if cylindrical $)=\pi(7 \mathrm{in} .)^{2}($ 5.5in. $) \approx 847 \mathrm{in}^{3} \approx 13.8 \mathrm{l}$

medium torus: torus radius $=6 "$ and diam of cross-section $=6 "$

medium volume (if toroidal) $=2 \pi 6 \mathrm{in} .\left(9 \pi i n .^{2}\right) \approx 1066 i n^{3} \approx 17.5 l$

large inflated dimensions: diameter $=62 \mathrm{~cm}$., thickness $=30 \mathrm{~cm}$.

large volume (if cylindrical) $\approx 90.5 l$

Note that two papers have been written which model the volume of a round mylar balloon with more accuracy than the simple model given here. ${ }^{2,3}$ Using their results we obtain the following estimates for the volume of the small and large balloons:

small volume $=1.2185 r^{3} \approx 1.2185(22.86 \mathrm{~cm})^{3} \approx 14.5 \mathrm{l}$

large volume $=1.2185 r^{3} \approx 1.2185(39.37 \mathrm{~cm})^{3} \approx 74.4 l$

Once the volume is estimated, and the weight of the empty mylar balloon bag is known, the 
lift can be estimated using Archimedes' principle and the densities of helium and air. (The following densities were used Helium $\approx 0.1786 \mathrm{~g} / \mathrm{l}$ Air $\approx 1.204 \mathrm{~kg} / \mathrm{m}^{3}$ ).

Un-inflated balloon masses:

○ large round $\approx 34.5 \mathrm{~g}$

$\circ$ medium toroid $\approx 18.5 \mathrm{~g}$

$\circ$ large round $\approx 13.5 \mathrm{~g}$

After the student teams performed this calculation the lift of the balloons was experimentally determined. This was done by determining the mass of clay necessary for the system to be neutrally buoyant.

Required additional mass for the balloon to be neutrally buoyant:

○ large round $\approx 42.3 g$

- medium toroid $\approx 0 g$

$\circ$ small round $\approx 3.9 g$

Interestingly, the medium toroidal balloon is neutrally buoyant and cannot provide any lift, thus it is basically useless for this project.

If the experimental results for the large and small balloon lift forces are converted back to volumes required to provide that lift we obtain:

$\circ$ large round volume $\approx 76.8 l$

- small round volume $\approx 17 l$

Thus the relative volume errors are as follows:

\begin{tabular}{r|cc} 
balloon & cylinder/torus & paper $^{2,3}$ \\
\hline large & $17.8 \%$ & $3.1 \%$ \\
medium & $5.4 \%$ & $\mathrm{n} / \mathrm{a}$ \\
small & $18.8 \%$ & $14.7 \%$
\end{tabular}

\section{Camera Calibration}

The end goal of the project requires a photograph of the test area which includes the entire figure in a single photo. Since the angular field of view of the camera lens is fixed, the size of the ground encompassed in the image can only be changed by changing the distance between the camera and the ground. The students were given the task to determine the angular field of view of their cameras by experimentation and analysis. They were given 2 weeks to come 


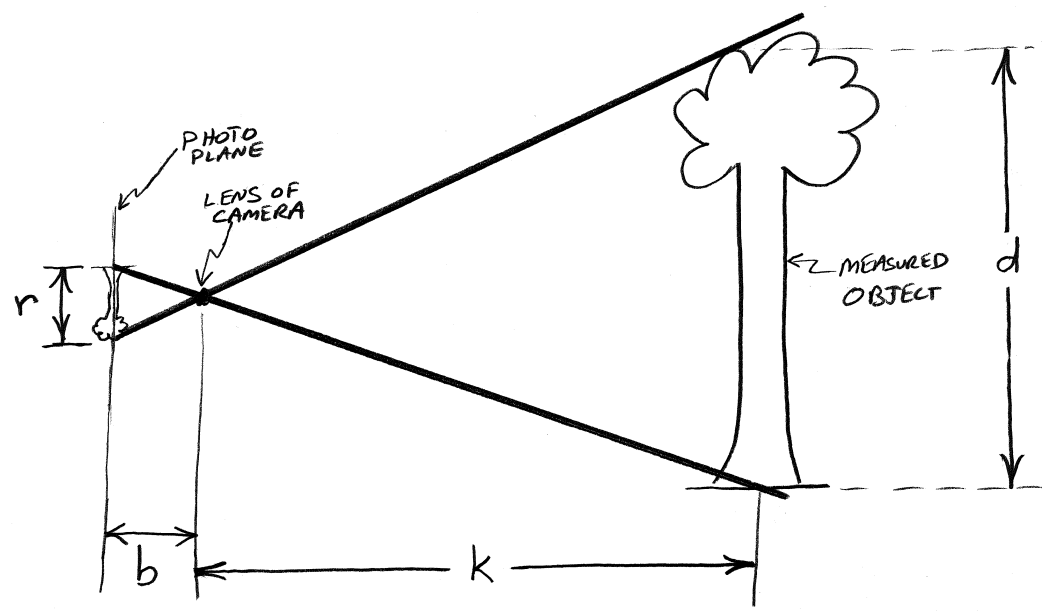

Figure 3: Help-sheet for camera calibration procedure

up with a calibration procedure to determine the angular field of view. A help sheet was handed out at the end of the 2 weeks to help them with the analysis of the calibration procedure, but it was up to them to finish the analysis, carry out their experiment, take the necessary measurements, and use those measurements to calibrate the camera. Since the bounds on the size of the test area are known, the upper and lower bounds for the required height of the camera could be determined (so that the entire test area can be photographed in a single photo).

\section{Height Determination}

Using the camera calibration from section 6 , the students could determine the actual size of objects in their photos if they knew the height of the camera when the photo was taken. The rules of the project did not allow the students to directly measure the length of the tether that they were using to hold onto their aerial platform. This was forbidden for two reasons, 1) the tether would most likely not be vertical or straight, so that length of the tether would not accurately represent the height of the platform, and 2) this provided an additional problem for the team to think about measurements and the effects of errors in those measurements. The students needed to devise a method to indirectly measure the platform's altitude. The most common method developed by the students was to measure a distance on the ground from the edge of the square test area to a single person holding the inclinometer. They used this distance (plus an average half distance of the upper and lower bounds of the side of the possible test areas, i.e. $+37.5 \mathrm{ft}$.). However, after they had developed their height determination procedures, we discussed the problems associated with 
making such a guess and the effect it would have on their estimated height measurement. They were asked to try and devise a procedure to estimate the height of the aerial platform which used 2 angle measurements and a distance measurement(not of the target area). Many of the teams were able to come up with a simplified version of the method outlined below, which several teams used during test day.

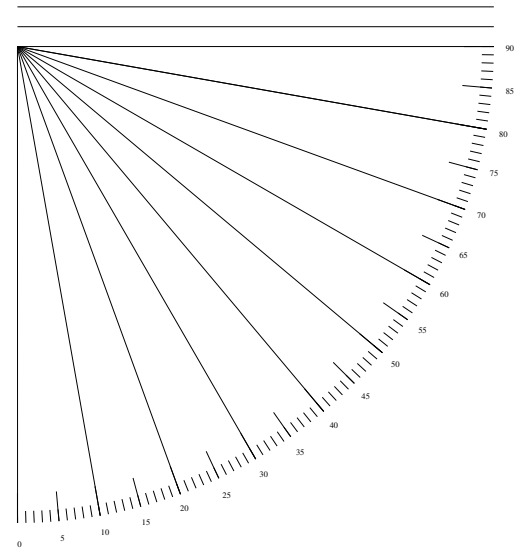

Figure 4: Angle paper distributed to students for height measurement

$$
\begin{array}{r}
s_{1}=h_{1} / \tan \left(\theta_{1}\right) \\
s_{2}=h_{2} / \tan \left(\theta_{2}\right) \\
l^{\prime}=l-s_{1}+s_{2} \\
z=\left(\tan \left(\theta_{1}\right) x_{1}\right. \\
z=\left(\tan \left(\theta_{2}\right) x_{2}\right. \\
x_{1}=x_{2}-l^{\prime} \\
\tan \left(\theta_{1}\right) x_{1}=\tan \left(\theta_{2}\right)\left(x_{1}+l^{\prime}\right) \\
z=\tan \left(\theta_{1}\right) \frac{l^{\prime} \tan \left(\theta_{2}\right)}{\tan \left(\theta_{1}\right)-\tan \left(\theta_{2}\right)}
\end{array}
$$

Distance between people $=25 \mathrm{ft}$.

Image 1: 44 degrees, 37 degrees (people's height: 5'9", 6’2")

Image 2: 31 degrees, 23 degrees (people's height: 5'9", 6'2")

Thus the heights of the camera when the image was taken are calculated to be $\sim 93.4 f t$. for image 1 and $\sim 43.3 f t$. for image 2 . 


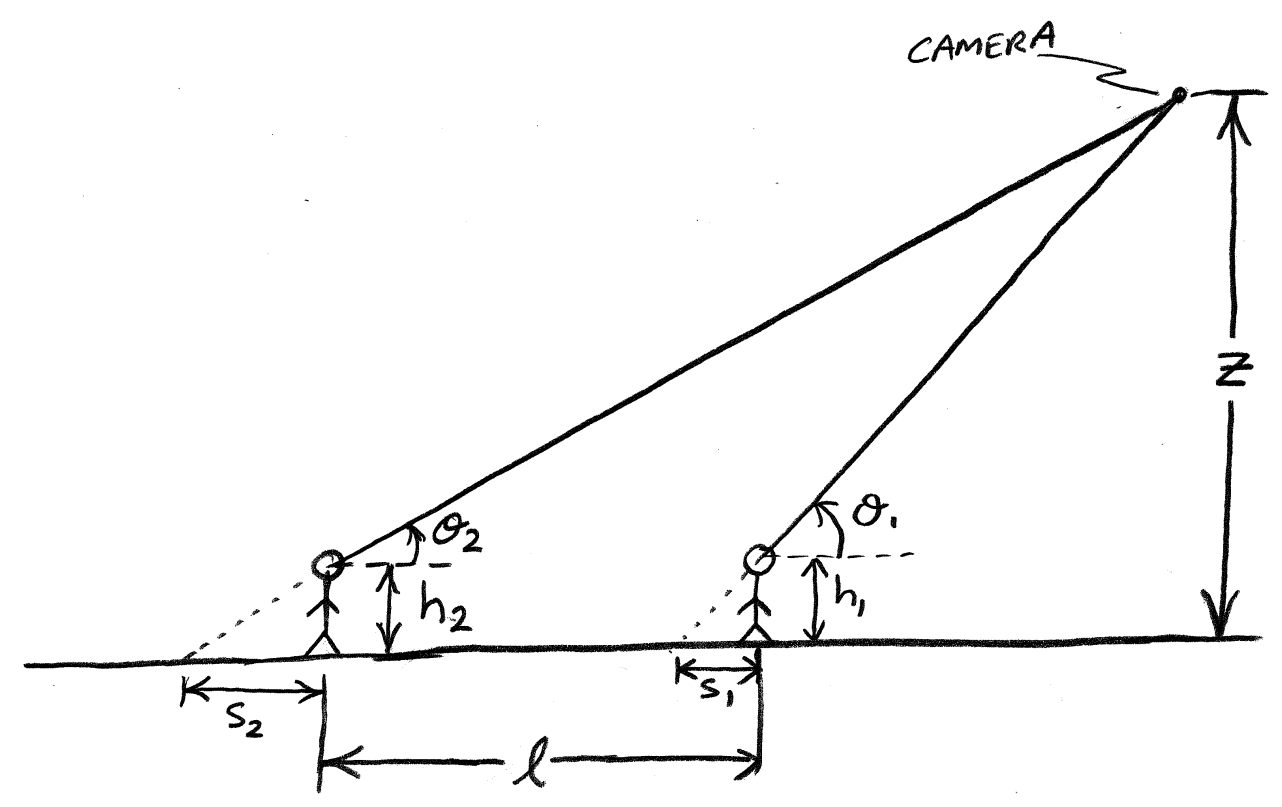

Figure 5: Method for determining the height of the camera

\section{Image Processing and MATLAB}

Once a week students had a 50 minute laboratory session where they learned the basics of how to write a computer program. The major concepts introduced were variable assignment, loops, if-then statements, and functions. After working through each fundamental concept and a session on practical debugging techniques with examples, they began work on the final image processing program. The program and functions were picked apart and each session a piece of the final program would be posed as a problem to be solved in teams during the session or to finish for homework. At the end of the course the final program and function files were put together into a single unit and used to calculate the area of the target image. The output from the program is an approximate area which is given in pixels ${ }^{2}$. A simple conversion from pixels length to distance on the photograph gives them the area of the target shape in distance squared on the photo. They then used this software program, their camera calibration data, and the height of the camera to determine the area within the target shape. The MATLAB program listing appears in Appendix A of this paper and is also available from the author upon request. 

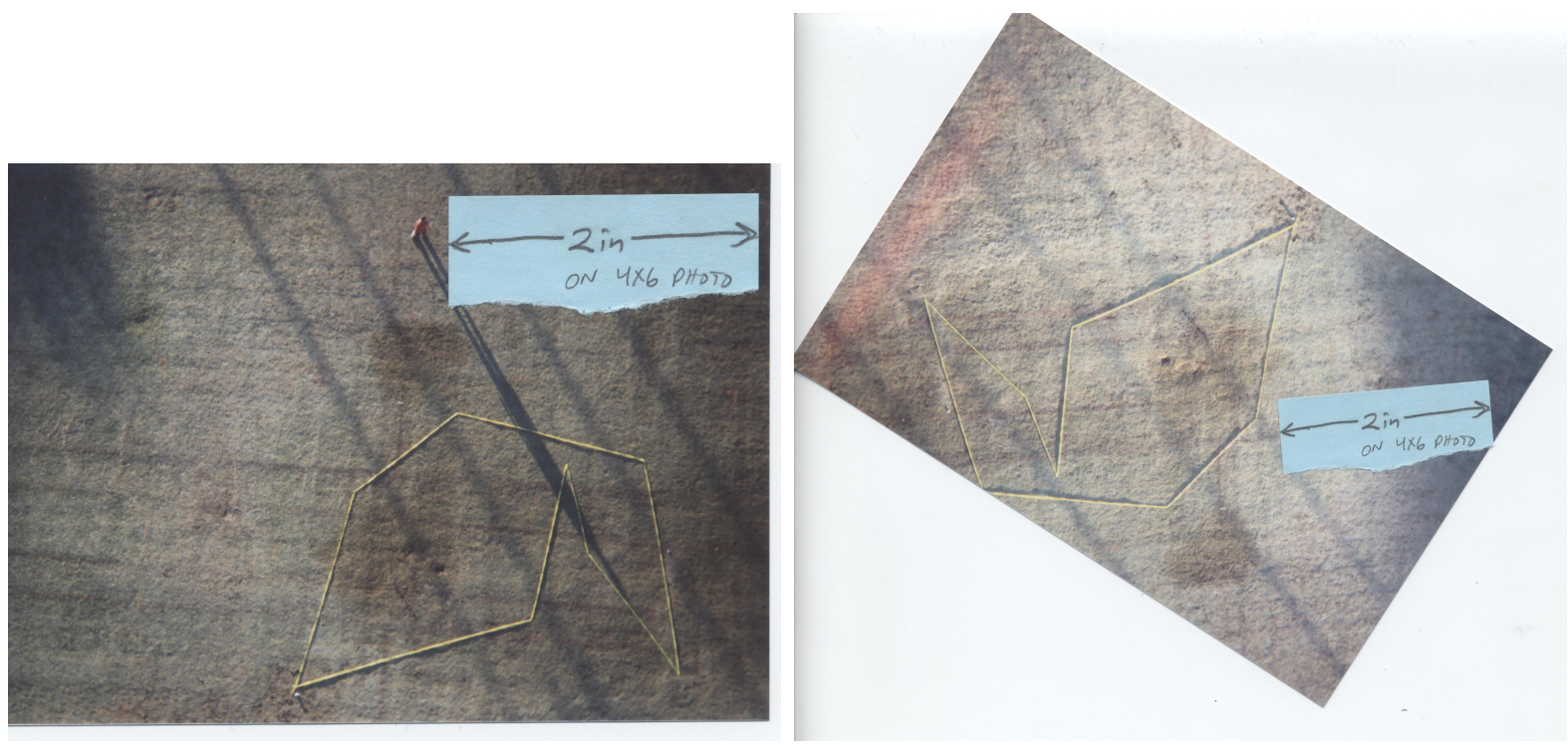

Figure 6: Photographs taken by an aerial camera showing one of the target areas

\section{Project timing}

The semester consisted of 16 weeks, with 1 hour lecture per week with all the students in the class, 1 hour MATLAB laboratory per week ( $\sim 30$ students per section), and a 1 hour recitation per week ( $\sim 30$ students per section). Two school holidays occurred resulting in 14 lectures during the semester. The first half of the course consisted of lectures and work on fundamental engineering principles (units, significant figures, length, volume, area, mass, force, pressure, hydro-statics, heat transfer, energy and power) and programming concepts using MATLAB (pseudo-code, variables, branching, conditionals, loops, arrays, plotting). The first project assignment was given as a part of the homework during week 8. Groups were formed by week $9 \& 10$ and lectures on team dynamics were given along with group activities in recitation for taking calibration photos. The design process was covered in week 11 with work on geometric optics done in recitation. Mathematical modeling was covered on week 12 to introduce simple mathematical models to help prepare them to model the volume of their balloons and then use their knowledge of hydro-statics to estimate the buoyancy force of each balloon. Empty balloon mass measurements were taken during recitation and guidance in creating their models was provided. Week 13 was a holiday for lecture, but groups met and worked together on their project that week. Week 14's lecture continued mathematical modeling and both lab and recitation were entirely devoted to in-class group work for the final project. Week 15's recitation class was devoted to each group taking photographs of the target area using their timer and chosen set of balloons. Week 16's lecture showed them how 


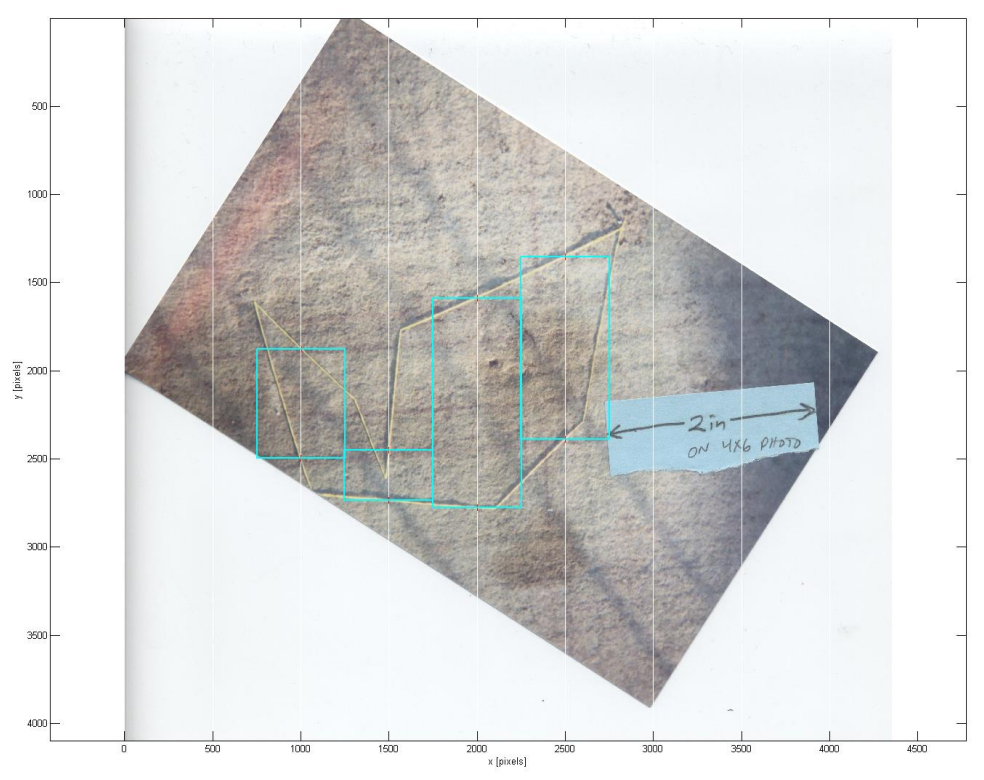

Figure 7: area $\sim 906 f t^{2}$

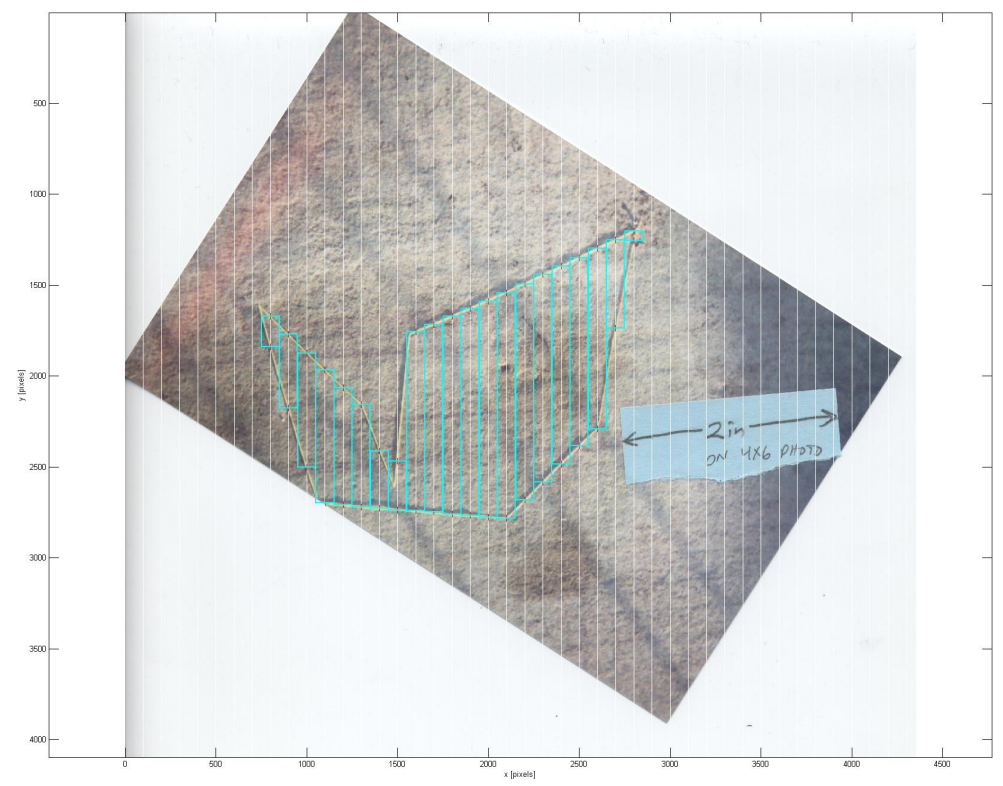

Figure 8: area $\sim 950 f t^{2}$

$$
\begin{aligned}
& 0 \\
& \stackrel{0}{0} \\
& \stackrel{D}{0} \\
& N \\
& \stackrel{N}{0} \\
& \vec{D} \\
& \stackrel{0}{D}
\end{aligned}
$$


to scan in their photograph so that the image file would work with the MATLAB program they had written in lab.

\section{Graded project assignments}

The project was broken up into segments which were included in the weekly homework assignments starting with homework 7 which was assigned during week 8 due a holiday occurring on week 3. Due to the presence of individual assignments as well as peer-evaluations at the end of the project, it was possible for members of the same team to receive different grades for the project component of the course. Major components of the final paper were developed in pieces each week using the project parts of the homework assignments given below.

\subsection{HW 7 (project part)}

(INDIVIDUAL ASSIGNMENT) As an individual brainstorm 2 different ideas for timer mechanisms which will trip the camera switch to take a photo. Turn in one sheet of paper with one idea per side of paper. Make a big sketch of your ideas and add clarifying labels and text explanations so that anyone looking at your paper can understand your idea.

\subsection{HW 8 (project part)}

a)(INDIVIDUAL ASSIGNMENT) Using the 90 degree angle protractor given to you (or printed out from blackboard) make a device which will measure the angle between your line of sight and the ground. (hint: gravity might be useful here in designing this device) You can make this angle measurement device using any materials you want (I suggest soda straws, string, cardboard, ...) Take a digital photo of your device and put that digital photo into a word processing program with a figure number and label describing the device and how it works.

b)(INDIVIDUAL ASSIGNMENT) Using your device taking as many angle measurements as you wish, but only one length measurement (using a tape measure, pacing off known foot lengths, etc.) Devise a way to calculate the height of the East Tower building on campus as accurately as you can. Calculate the height, remember to use significant figures.

c) (CHALLENGE) optional Get accurate narrow error bounds on your answer in problem \#3) above. 


\subsection{HW9 (project part)}

(TEAM ASSIGNMENT) (Turn only one of these in per team, put your team name-number as well as all the team member's names on the assignment)

a) Turn in 2 different ideas for getting your camera up in the air to the height you think you will need. Each idea must have a detailed sketch and a short, but complete explanation of how it will work

b) Turn in 3 different ideas for autonomously triggering your camera (note: rule amendment, you are not allowed to use the tether string in anyway for triggering your cameras) Again, each idea must have a detailed sketch and a short, but complete explanation of how it will work.

c) For each of the 5 ideas, as a group list all of the things that you like or dislike about it (i.e. which ones are more risky, what are the advantages of this idea, what could go wrong, what aspect of the design are you sure will work)

\subsection{HW10 (project part)}

(TEAM ASSIGNMENT):(turn in 1 set per group)

1)Make a group decision about what method you will focus on for your up \& trigger mechanism. Turn a group decision matrix for each (1 for up, and 1 for trigger)

2) Take your calibration photos. Take at least 3 photos of a known length(s)[up to you] from a known distance away(10ft to 30ft). Take all your pictures on the ground (i.e. no need to get your camera up in the air to do this). NOTE: keep a log of the photo number, brief description of what your a photographing, and the measured distance, and known length for each photo, you will need this later. Pick the best one and go to computing commons or somewhere that has a scanner and scan it in so you can import it into a word processor, print it out and turn in the printout.

3) Take a few(3-4) photos of "unknown" lengths(record the length anyway) that are a known(i.e. measured distance away). (go to computing commons or somewhere that has a scanner and scan in 2 of these, import to word processor, print out and turn in.)

4) In a MEMO(see HW3) describe how you will use your calibration photo to determine the "unknown" lengths, do your calculations on the 2 photos in part 3) and see how close you were. 


\subsection{HW11 (project part)}

(TEAM ASSIGNMENT): (turn in 1 set per group)

1) Think of a way to measure/estimate the volume of the small, medium, and large balloons. Clearly describe your method. Carry out your method to obtain the volumes of the 3 balloons when inflated. Try to bound your answer(i.e. it should be no greater than this number and no less than this number)

2) Using Archimedes's principle (see Moaveni to remind yourself if needed) calculate the lift force that the small balloon will provide when inflated with helium. (NOTE: you are not allowed to measure this directly, i.e. by filling the balloon with helium and measuring it somehow). What assumptions are in your model, what things are important?

3) Clearly document your method for determining the height of your camera when it takes the picture. Using a spreadsheet create a table with 2 parameters(i.e. things you measure) one parameter across the top, and one down the side. Fill the table with the corresponding heights so that you can quickly determine the height of the camera once you know the 2 measurements that you take. If your method requires more than 2 measurements think of ways to either reduce it to 2 , or to use those multiple measurements so that it will be possible to quickly estimate the height from the measurements.

4) Using your calibration photos, clearly explain what height range your camera needs to reach in order for it to take a single photo which contains the entire test area.

5) How many balloons of which type would you need to lift your current trigger mechanism? (or if you are not using balloons as your primary lift system, how many would you need if you are using them for the back-up system?).

\subsection{HW12 (project part)}

(TEAM ASSIGNMENT): (turn in 1 set per group) Get started on your final project writeup. Turn in your best current draft of sections $2,3,4$, and 8 from the outline. These are a cleaned up version of the items that you have already turned in for hw previously. So what you turn in should be neat and understandable.

\subsection{FINAL EXAM}

In place of a final examination, each team of students presented a Power Point Presentation of their design, analysis, and results along with a written report. 
Note that the outline which was provided for the students describing what was expected for the final paper and the final presentation is included in Appendix B of this paper.

\section{Informal Evaluation}

Although no formal formative or summative evaluations of the project design was done at the time of implementation, comments in the course evaluations addressed the project and provide insight into ways to improve the project. A majority of the students in the class spent from four to eight hours per week studying and working on the course material. Anecdotal evidence suggests that more hours were spent in the latter half of the semester working on the final project than in the first half of the semester. Some of the free-responses to the following questions addressed the course final project. The responses were to the following questions, "What did you like most about this course?", "What did you like least about this course?", and "Comments". Several students stated that they "enjoyed" the project with reasons that fell into the following categories: 1) it was hands-on 2) incorporated much of what they learned in class 3) group interaction 4) stimulated creative thinking (few restrictions) 5) learning MATLAB 6) challenging. Several students also made suggestions for improving the course which fell into the following categories: 1)MATLAB was way too hard! 2)MATLAB moved too fast 3)Too much pressure on final project 4)Final project weighted too high 5)Did not like my project group 6) Difficult to schedule outside group time.

Although the comments from the informal project evaluation provide some guidance in improving the project, a more formal evaluation relating specifically to the design project would have provided a much better basis for project improvement.

\section{Troubleshooting}

The first year that this project was run the weather was extremely favorable on test day with little or no wind. However the second year we were not so fortunate with weather. Although we had no rain, it was extremely windy throughout the day. This caused major problems for students trying to obtain photographs of the target area. several teams were able to obtain photographs of the target area but the vast majority of students were not, either due to the weather conditions, or to their trigger design, or to their lack of measuring the camera height, or to not all of the team members being able to show up for their allotted time. Thus, in order that they be able to carry out the rest of the project, I and one of the teaching assistants and a friend, used our own simple trigger design and camera to obtained some images of the target area with the associated altitude measurements (see Fig. 7). These photographs and measurements are then distributed to the entire class to be used in 

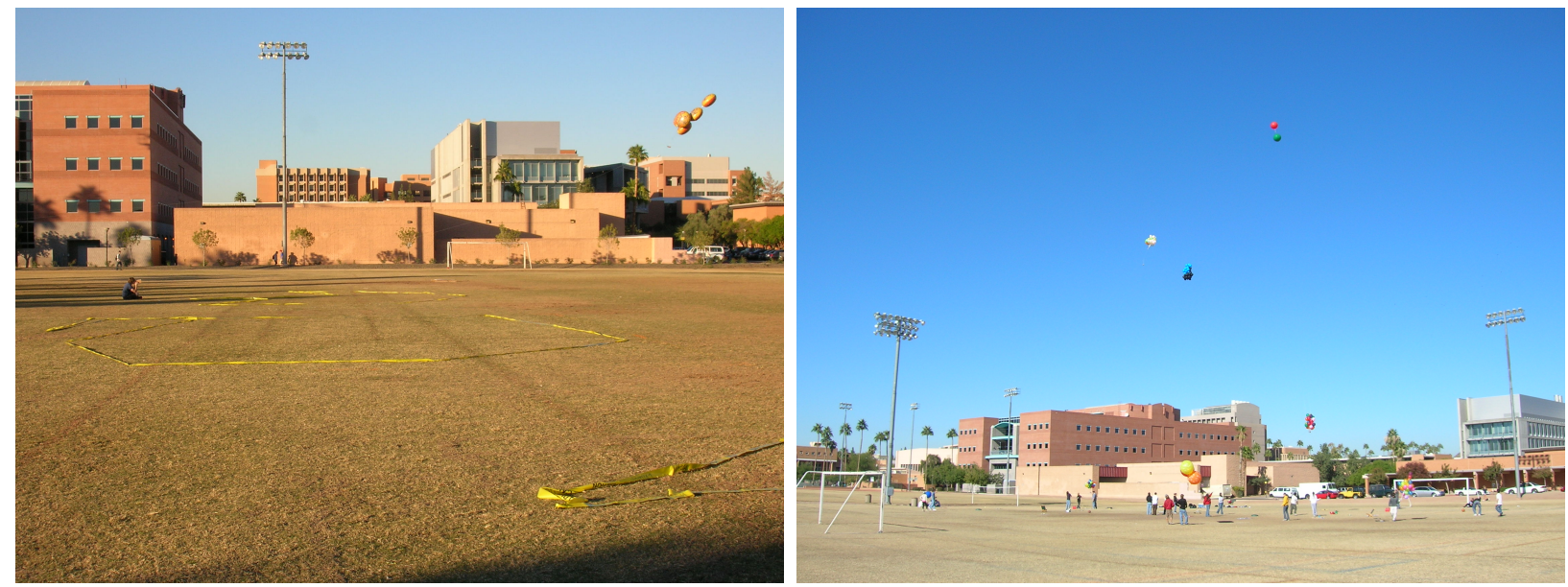

Figure 9: Example of a student group taking photographs and data on a test day with high wind speeds and an example of many student groups taking photos on a test day with very low wind speeds.

the analysis if their team was unable to obtain the required photographs and data.

The general instructional approach used was to discuss some tools which could be used to solve a particular problem and then let the students work on problem and let them devise their own solutions. Then after they have attempted the problem and turned that attempt in, we would discuss some possible approaches which might be successful. This approach allowed for creativity and self-exploration. Some students enjoyed this open-ended opportunity but other students did not.

\section{Conclusions and Proposed Changes}

This is a rather complex project with many logistical aspects and instructional challenges that need to be overcome to execute it successfully. As with any project there are improvements that could be made to improve the efficiency and efficacy of the project.

The MATLAB component although broken apart into several pieces, was rather ambitious, given that this in an introductory course which assumes no programming background. The in-class work in lab had several MATLAB programming problems each week, with the later weeks leading to building the program in sections. Although each group had a functioning MATLAB code at the end of the course, it is very possible that several individual team members had difficulty integrating the pieces and instead relied on their teammates for the final integration. In the future, I would expand the MATLAB assignments in place of some 
of the traditional course based HW problems.

Over $75 \%$ of the teams used the mechanical hour timer in their final designs. Removing the mechanical hour timer from the kit would be a significant improvement, since students would then need to design, construct, and calibrate their own timers. However, designing a reliable mechanical timer and trigger mechanism from only the provided materials is a challenging task in itself for many students (as well as instructors), especially in the time constraints of the project.

This project had many components and each component could have been explored in much more depth. Although that was part of the original design intent, so that teams who just wanted to do the minimal amount could succeed and those that wished to explore a particular part in more depth could also do so, at times it felt like there were too many pieces that needed to come together. I feel that segmenting each problem further would improve the project. The students had the most difficult time with the camera calibration step of this project. Thus either the camera calibration step could easily be removed to allow more time for other aspects of the project. Or more time could be spent on the camera calibration step, exploring both error propagation and the accuracy of the assumptions used for that analysis.

Because weather is unpredictable, a "rain" or "wind" date would be a very good idea. Unfortunately with a large class and the scheduled rental of helium tanks, this was deemed not possible. Overall, I would not recommend this project be attempted with such a large number of students $(\sim 45$ teams of $3-5$ students/team) as the logistical issues were quite difficult to overcome.

Various components of the project provide many interesting and approachable challenges for students of many different levels of interest and ability.

\section{Acknowledgements}

The author would like to thank Dr. Praveen Shankar for the ballon volume references and for assistance in conducting the project. The author would also like to thank Lawrence Duff, Andre Magdelano, and Leonard Bucholtz for supervising and assisting the students in constructing their mechanical timer devices. Thanks also to Dr. Bruce Steele for helpful conversations about this project design and help with providing instrumentation. 


\section{References}

[1] Susan A. Ambrose and Cristina H. Amon, Systematic design of a first-year mechanical engineering course at carnegie mellon university, Journal of Engineering Education 86 (1997), 173 -182 .

[2] Ivailo M. Mladenov and John Oprea, The mylar baloon revisited, The American Mathematical Monthly 110 (Nov. 2003), no. 9, $761-784$.

[3] William H. Paulsen, What is the shape of a mylar balloon?, The American Mathematical Monthly 101 (Dec. 1994), no. 10, 953 - 958. 


\section{Appendix A}

Main program file for the image processing program which students wrote in section after learning programming basics such as variable assignment, loops, and if-then statements.

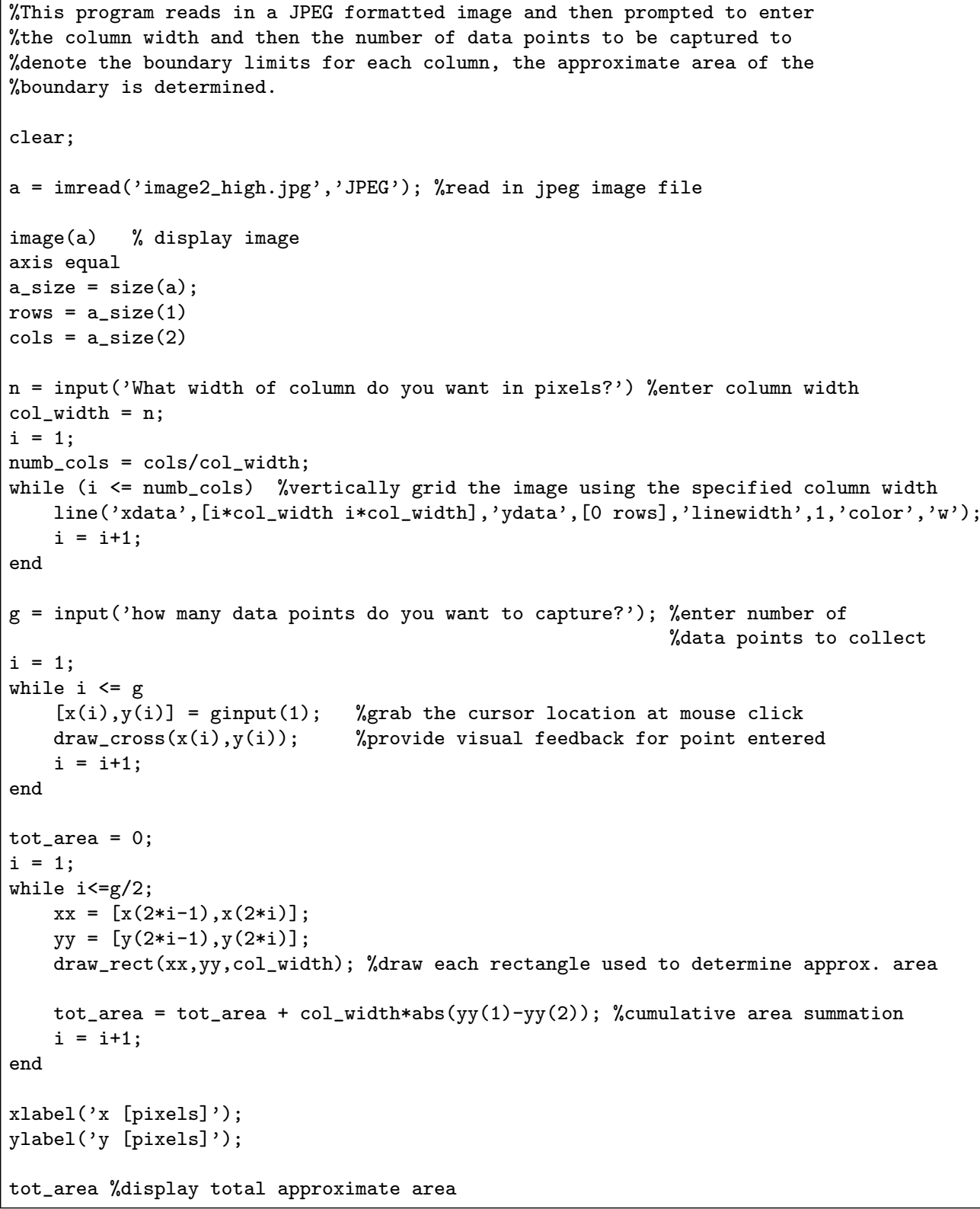


draw_cross.m: function file for drawing the cross-hairs on points clicked by the user

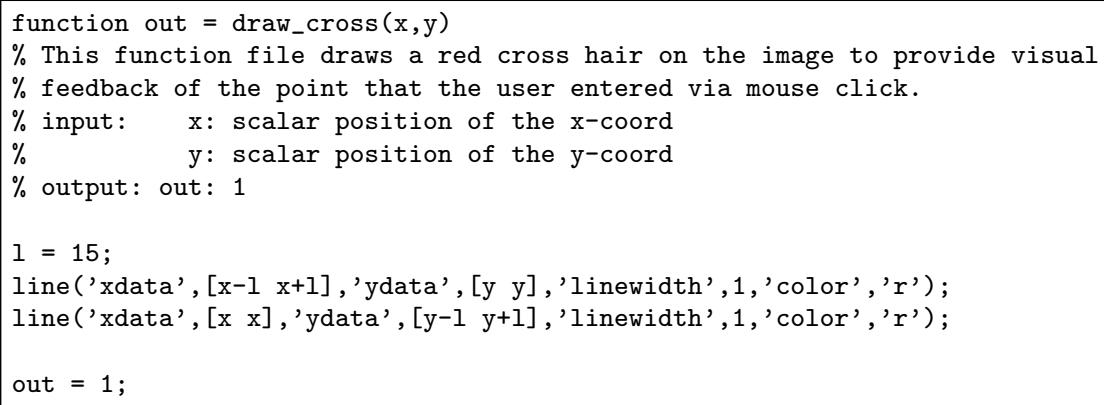

draw_rect.m: function file for drawing the rectangles corresponding to the user's input clickpoints to approximate the enclosed area

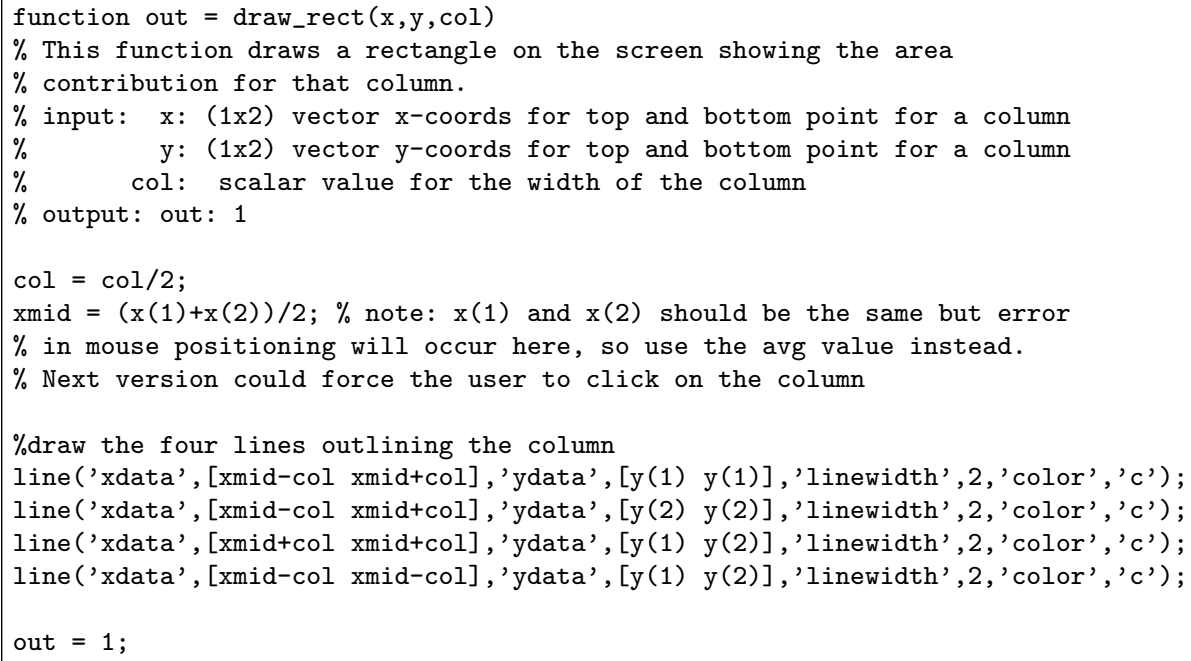

\section{Appendix B}

\section{Outline for Final Project Write-Up}

Must be typed, double spaced, no smaller than 14 point font. All photo scans should scaled so that they appear in the paper actual size. The final report is due on the last day of class at the beginning of lecture, December 3rd. Note that many of the parts of the report can be completed before you take your photos on test day. You will need to do this since for many of you your test photos will be taken on the Friday before the report is due.

1. Abstract The purpose of the abstract is to briefly describe the goal of your project. It should include the constraints and resources with which you were required to operate. The specific results of your analysis should also be included. If someone were to only read this 
section, they should have a reasonable idea of what you did, and what you found out. This section can be no longer than 1 page. Be as descriptive as possible while also being as brief as possible. This must be the absolute last section that you write and will require a few revisions to get it right (specific but brief).

2. Specifications: Give the specific specifications which you had to design within. What were you given, what were your constraints.

3. Designs Explain each of the preliminary designs that you considered for your lift and trigger mechanisms. Include sketches(can be scanned in or xeroxed in hand sketches if done neatly) along with figure numbers and captions under each figure. The captions must be descriptive

(GOOD!!!. Figure 3: The slingshot lift mechanism where the rubber balloons are stretched by the user and then released which launches the camera into the air over the target area)

(NOT!!!! Figure 3: lift mechanism 2)

Include in this section your group decision matrix on lift and trigger mechanisms. Remember that your each row will correspond to each of the exploratory designs that you discussed earlier in this section and each column will be an important attribute(note that you MUST describe each attribute so that the reader completely understands what you mean). (i.e. if an attribute is simplicity do you mean simple design, simple to build, simple to operate, etc.) Lastly, describe your final design in words, pictures, and drawings. Describe the reasons for choosing it. If it differed from your choice in your decision matrix, describe the reasons for the change. What redesigns were required to get it working.

\section{Methods}

Describe your complete method for determining actual lengths in the target area from measured lengths in your photographs. Include a sketch of your method along with a scan of your calibration photo(you have access to scanners in computing commons, ask the helpdesk there for help operating the scanners if you need it). Include scans of any measurement devices that you used. Describe the plan you implemented for each person so that all the tasks required are completed during the 15 minutes on test day. Make a reference to the spreadsheet height estimation table which is included in your appendix.

Describe your method for calculating the area of the irregularly shaped drawing in the target area. Include hand sketches if they help to clarify your method.. Give a brief overview of your computer program, describe the method that it uses to calculate the area. In no more than $1 / 2$ page give a brief pseudo-code structure of your program. Make a reference here for a complete listing of your program which is included in your appendix.

5. Data 
This section includes all of your data. In this case that means scans of the 3 best photos that you took on test day along with the measurements that you took to determine the heights for each of those photos. Again, make sure that you include descriptive figure captions for every figure in this section. Include in the appendix scans of every photograph you took on test day along with the measurements taken.

\section{Analysis/Discussion}

Work through the analysis for the target area side length. Describe the advantages or disadvantages of the method that you developed. How accurately could you make each measurement? Do larger relative errors in some measurements have a larger impact on your answer than others (i.e. if one measurement is $5 \%$ off will that change your answer more or less than if a different measurement if off by $5 \%$ ?).

Give a table of results for the area of the irregular shape. Run your program to analyze your chosen photograph at least 8 times with different column widths. The entries in the table are your column width and the resulting approximate area. Decrease your column width several times until your resulting area approaches a constant value. Include an image of the matlab figure file showing your image with the digitized points and the rectangles.(include your column width for that image in your figure caption)

What are all the sources of error in your measurements and analysis? Which ones do you feel are the most dominant sources of error? Give reasons for your choices. What are the tightest bounds that you feel the length of the side of the target area falls within? What are the tightest bounds that you feel the area of the irregular area falls between? Use the numbers from your analysis to support your choice of bounds.

\section{References}

The purpose of the references is to document all of the sources of information that you used. A list of all books, articles, websites, people, etc. that you used.

www.apastyle.org is a useful resource for referencing your sources

\section{Appendix}

A. Spreadsheet used for rough height estimation

B. MATLAB Program listing

C. All test-day photos with measurements taken for each.

D. anything else you feel needs to be included in the appendix.

Power-Point Presentation: (each group will have about 10mins to present and 2 mins for questions) The presentation will be given during the time for the final exam during exam 
week.

1. Slides should be simple with no annoying and distracting transitions

2. Use either a dark background with light text or a light background with dark text. All text should be easily readable.

3. Allow about 1-2min. per slide

4. No slide should have a large amount of text on it.

5. All images should be as large as possible, i.e. take up as much room on the slide as possible.

6. Practice your presentation as a group a few times beforehand. Have someone in the group count the number of times, and raise their hand every time, the speaker says the word "Um.." or a similar word.

7. Everyone in the group needs to present approximately an equal part of the talk

8. Speak slowly and clearly so that everyone in the class can hear you, if you find yourself talking quickly when you are nervous, make a conscious effort to watch your pacing and slow yourself down.

9. Your first slide must be a tittle slide with your group name, individual names, class title, semester and year. You can put a photo of your group/project behind the text.

10. Be professional. 